\title{
Pandemia COVID-19 e Ações do Núcleo Ampliado de Saúde da Família e Atenção Básica na Rede SUS
}

\author{
Otamares Castro Simões Coelho, Anelisa Terrola Martins Ferreira, Raquel de \\ Deus Mendonça
}

\section{RESUMO}

O Núcleo Ampliado de Saúde da Família e Atenção Básica (NASF-AB) visa ampliar as ações desenvolvidas pela Estratégia de Saúde da Família. Entretanto, suas atividades sofreram alterações temporárias, devido à pandemia da COVID-19. O objetivo deste trabalho é descrever as atividades e serviços realizados pelos profissionais do NASF-AB de uma regional na rede SUS-BH durante a pandemia de SARS-CoV-2. Trata-se de um estudo transversal com amostra de conveniência em Belo Horizonte-MG. Os dados foram coletados por um questionário online autoaplicado. Participaram 38 profissionais, dos quais 71\% atuavam na Atenção Básica há mais de 5 anos. $\mathrm{Na}$ pandemia, observou-se falta de acesso a treinamentos (71\%) e equipamentos tecnológicos (53\%) para acompanhamento dos usuários. Os grupos operativos foram $100 \%$ suspensos e $90 \%$ relataram acesso aos Equipamentos de Proteção Individual. Apesar dos desafios que dificultaram a reorganização dos trabalhos e o acompanhamento dos usuários durante eventos excepcionais, o NASF-AB apresenta-se como uma potente estratégia de cuidado.

Palavras-chave: Atenção Primária à Saúde; Avaliação de Programas e Projetos de Saúde; Infecção por Coronavírus; Pandemias.

\section{ABSTRACT}

The Expanded Health Center of Primary Care and Family (NASF-AB) aims to expand the actions developed by the Family Health Strategy. However, due to the COVID-19 pandemic issues, activities suffered temporary changes. The objective of this research was to describe the activities and services performed by professionals at the NASF-AB in a region in the SUS-BH during the SARS-CoV-2 pandemic. It was a cross-sectional trial with a convenience sample in Belo Horizonte-MG. Data was collected online self aplied. 38 employees were evaluated, of which $\mathbf{7 1 \%}$ worked in Primary Care for more than 5 years. In this pandemic, there was a lack of employee training (71\%) and access to technological equipment (53\%) for monitoring users. The operative groups were $100 \%$ suspended, and $90 \%$ of professionals reported access to Personal Protective Equipment. Despite the challenges that made it difficult to reorganize work and monitor users during exceptional events, the NASF-AB presents as an excellent care strategy.

Keywords: Primary health care; evaluation of health programs and projects; health personnel; Infection from coronavirus; Pandemics.

Submissão recebida em 28 de julho de 2021.

Aceito para publicação em 09 de dezembro de 2021.

Avaliado pelo sistema Double Blind Review

Revista da Rede APS 2021

Publicada em: 28/12/2021

DOI:10.14295/aps.v3i3.199

Otamares Castro Simões Coelho (Secretaria Municipal de Saúde, Prefeitura de Belo Horizonte, Belo Horizonte, MG, Brasil)

Anelisa Terrola Martins Ferreira (Secretaria Municipal de Saúde, Prefeitura de Belo Horizonte, Belo Horizonte, MG, Brasil)

Raquel de Deus Mendonça (Universidade Federal de Ouro Preto, Ouro Preto, MG, Brasil)

Correspondência para:

Otamares Castro Simões Coelho otamaresdecastro@hotmail.com 


\section{INTRODUÇÃO}

A pandemia do SARS-CoV-2 é um dos maiores desafios sanitários em escala global deste século (WERNECK; CARVALHO, 2020). A COVID19 trata-se de uma doença infecciosa e altamente contagiosa, declarada como pandemia em março de 2020 pela Organização Mundial de Saúde (OMS) (OMS, 2020). No Brasil, até o dia 15 de julho de 2021 havia 19.209.729 casos acumulados e 537.394 mortes (BRASIL, 2021).

O enfrentamento da pandemia é considerado função essencial da Saúde Pública através de ações voltadas para a população (FILHO et al., 2020). No Brasil, são muitos os desafios, devido às características de transmissão em circunstâncias de grande desigualdade social, condições precárias de habitação, saneamento básico, entre outros (WERNECK; CARVALHO, 2020).

Dessa forma, a epidemia do SARS-CoV-2 depara-se com a existência de múltiplas vulnerabilidades na população brasileira, associado a altas taxas de desemprego, cortes nas políticas sociais e subfinanciamento do SUS (MATTA et al., 2021). Nos últimos anos, após a aprovação da Emenda Constitucional no 95, que impõe radical teto de gastos públicos e com as políticas econômicas implantadas, há um crescente e intenso estrangulamento dos investimentos em saúde e pesquisa (WERNECK; CARVALHO, 2020;).

Para atuar na pandemia, os serviços de saúde precisaram reorganizar o processo de trabalho. Na Atenção Básica ( $A B)$, com a evolução do SARS-CoV-2, medidas foram tomadas para garantir $\mathrm{o}$ atendimento de qualidade das equipes do NASF-AB e reduzir o contágio pelo SARS-CoV-2.

O NASF-AB tem o objetivo de apoiar e ampliar a resolutividade na Atenção Básica (BRASIL, 2008). As equipes executam suas atividades em territórios definidos, sendo o acesso às ações oferecidas intermediadas pelos profissionais das equipes de Saúde da Família (MACIEL et al., 2015). A pandemia do SARS-CoV-2, entretanto, provocou alterações nas atividades e serviços do NASF-AB em relação à rotina habitual (RIBEIRO et al., 2020; OLIVEIRA et al., 2020; FERNANDEZ et al., 2020). Inicialmente foram suspensos algumas atividades e serviços como os atendimentos eletivos, visitas domiciliares, atividades coletivas, a exemplo: Lian Gong em 18 Terapias, grupos operativos, ações educativas e as reuniões de matriciamento (BRASIL, 2014). Os profissionais permaneceram nas Unidades Básicas de Saúde (UBS) em período integral de trabalho prestando apoio para as equipes de Saúde da Família (eSF).

Contudo, o NASF-AB tem novos desafios para sua atuação diante da complexa realidade ocasionada pela pandemia. Entre eles a garantia de acesso aos sistemas de saúde e as condições que possibilitem o autocuidado e a manutenção do estado de saúde dos indivíduos (RAFAEL et al., 2020). Diante do exposto, o objetivo deste trabalho foi descrever as atividades e serviços realizados pelos profissionais do NASF-AB na rede SUS-Belo Horizonte (SUS-BH) durante a pandemia de SARS-CoV-2.

\section{METODOLOGIA}

Trata-se de um estudo transversal com amostra de conveniência. $O$ estudo foi realizado na cidade de Belo Horizonte que possui um sistema municipal de saúde dividido em nove distritos sanitários. A pesquisa foi realizada com os profissionais do NASF-AB de um dos distritos sanitários.

Para a realização da pesquisa, foram considerados critérios de inclusão: ser profissional do distrito sanitário estudado, pertencer à equipe NASF-AB e concordar com o Termo de Consentimento Livre e Esclarecido (TCLE) e sua assinatura eletrônica. Como critério de exclusão foi considerado aquele que após quatro tentativas não respondeu ao questionário.

O estudo foi realizado em uma única etapa, no período de setembro a novembro de 2020. Os participantes receberam por e-mail, enviado pela Referência Técnica da regional e/ou 
gerente da UBS um convite e link de acesso ao questionário de coleta de dados, autoaplicado e confidencial, disponibilizado em plataforma online (Google Forms).

A cada semana a Referência Técnica e/ou gerentes reenviavam o e-mail com link da pesquisa. Além disso, foi realizado um estudo piloto com profissionais do NASF-AB e residentes multiprofissionais de saúde não atuantes na regional do estudo, com objetivo de avaliar e analisar a aplicabilidade do questionário e corrigir possíveis erros.

As variáveis sociodemográficas investigadas foram idade (anos), sexo, estado civil (solteiro, casado/união estável, viúvo, divorciado/separado), ocupação profissional (assistente social, farmacêutico, fisioterapeuta, fonoaudiólogo, nutricionista, profissional de educação física, psicólogo, terapeuta ocupacional), escolaridade (graduação, pósgraduação/especialização, mestrado, doutorado), tempo de atuação na atenção básica ( 0 a 5 anos, mais de 5 anos).

Para obtenção das condições de trabalho perguntou-se sobre: realização do teste para COVID-19 (sim/não), resultado do teste (positivo, negativo, não realizei o teste), contato com casos de COVID-19 (caso confirmado, caso suspeito, não tive contato), carga horária de trabalho (manteve, diminuiu, aumentou), local de exercícios das atividades laborais (UBS, homeoffice, ambos), distanciamento dos familiares (sim/não), treinamento para atuar na linha de frente ( $\operatorname{sim} /$ não), acesso às notas técnicas da Secretaria Municipal de Saúde (SMSA-BH) ( $\operatorname{sim} /$ não) e leitura das notas técnicas da SMSA$\mathrm{BH}$ (sim/não).

Além disso, investigou-se o acesso aos Equipamentos de Proteção Individual (EPI's) baseado nas recomendações da OMS, bem como a atuação durante a pandemia, a saber: atendimento individual, atendimento compartilhado, visita domiciliar, grupos operativos, reuniões de matriciamento, elaboração de materiais de apoio, rotinas, protocolos e outras ações de educação permanente. E perguntou-se quais meios de comunicação seriam ideais para aconselhar/orientar os usuários durante a pandemia (atendimento presencial, materiais impressos, vídeos, telefone, e-mail, whatsapp, redes sociais).

As análises dos dados foram realizadas com auxílio do software Data Analysis and Statistical Software (STATA) versão 13.0. Realizou-se o cálculo das frequências absolutas e relativas, médias e desvios-padrão.

O presente estudo foi aprovado pelo Comitê de Ética em Pesquisa da Gerência de Educação e Saúde da Secretaria Municipal de Saúde de Belo Horizonte (4.223.199) e do Hospital Metropolitano Odilon Behrens (4.217.490).

\section{RESULTADOS}

Participaram da pesquisa 38 profissionais de saúde que representam $52,77 \%$ do total $(n=72)$, com média de idade de 38 anos. Com relação aos respondentes, $92,11 \%$ eram do sexo feminino (Tabela 1).

Todas as categorias profissionais elencadas na equipe do NASF-AB foram representadas, com exceção do serviço social. A maior participação foi de nutricionistas (23,68\%), seguido por fisioterapeutas (18,42\%). Em relação à qualificação profissional, 78,95\% dos profissionais afirmaram possuir especialização e $71,05 \%$ relataram mais de cinco anos de vinculação à Atenção Básica (Tabela 1). 
Tabela 1. Caracterização sociodemográfica e formação dos profissionais. Belo Horizonte, 2020.

\begin{tabular}{|c|c|c|}
\hline \multirow[t]{2}{*}{ Características } & \multicolumn{2}{|c|}{ Total $(\mathrm{N}=38)$} \\
\hline & $\mathbf{n}$ & Valores \\
\hline \multicolumn{3}{|l|}{ Sexo (\%) } \\
\hline Masculino & 3 & 7,89 \\
\hline Feminino & 35 & 92,11 \\
\hline Idade (anos) & 38 & $38,74 \pm 10,20$ \\
\hline \multicolumn{3}{|l|}{ Estado civil (\%) } \\
\hline Casado(a)/União estável & 18 & 47,37 \\
\hline Solteiro(a) & 15 & 39,47 \\
\hline Divorciado(a)/Separado(a) & 5 & 13,16 \\
\hline \multicolumn{3}{|l|}{ Profissão (\%) } \\
\hline Nutricionista & 9 & 23,68 \\
\hline Fisioterapeuta & 7 & 18,42 \\
\hline Profissional de Educação Física & 6 & 15,79 \\
\hline Farmacêutica(o) & 5 & 13,16 \\
\hline Fonoaudióloga(o) & 5 & 13,16 \\
\hline Psicólogo (a) & 4 & 10,53 \\
\hline Terapeuta Ocupacional & 2 & 5,26 \\
\hline \multicolumn{3}{|l|}{ Escolaridade (\%) } \\
\hline Graduação & 5 & 13,16 \\
\hline Especialização & 30 & 78,95 \\
\hline Mestrado & 2 & 5,26 \\
\hline Doutorado & 1 & 2,63 \\
\hline \multicolumn{3}{|l|}{ Tempo de atuação na $A B^{*}(\%)$} \\
\hline 0 a 5 anos & 11 & 28,95 \\
\hline Mais de cinco anos & 27 & 71,05 \\
\hline
\end{tabular}

Fonte: Elaborado pelas autoras.

*AB: Atenção Básica

Em relação às condições de trabalho durante a pandemia; $78,95 \%$ dos profissionais realizaram o teste para SARS-CoV-2, sendo que $2,6 \%$ testaram positivo; adicionalmente $39,47 \%$ relataram contato com casos confirmados ou suspeitos no local de trabalho. A maioria relatou que a carga horária de trabalho se manteve sem mudanças, sendo que $97,37 \%$ continuaram exercendo suas atividades laborais no ambiente de trabalho e $86,4 \%$ afirmaram terem se afastado de seus familiares (Tabela 2).

Além disso, $71,05 \%$ relataram não ter participado de treinamentos para atuar durante a pandemia, mas $100 \%$ informaram que tinham acesso às Notas Técnicas da SMSA$\mathrm{BH}$ e a maioria relatou ler periodicamente (Tabela 2). 
Tabela 2. Condições de Trabalho. Belo Horizonte, 2020.

\begin{tabular}{|c|c|c|}
\hline \multirow[t]{2}{*}{ Características } & \multicolumn{2}{|c|}{ Total $(\mathrm{N}=38)$} \\
\hline & $\mathbf{n}$ & $\%$ \\
\hline \multicolumn{3}{|l|}{ Teste para SARS-CoV-2 } \\
\hline Sim & 30 & 78,95 \\
\hline Não & 8 & 21,05 \\
\hline \multicolumn{3}{|c|}{ Infecção por SARS-CoV-2 entre os que realizaram o teste* } \\
\hline Positivo & 1 & 3,33 \\
\hline Negativo & 29 & 96,67 \\
\hline \multicolumn{3}{|c|}{ Contato no local de trabalho com pessoas com SARSCoV-2 } \\
\hline Confirmado & 13 & 34,21 \\
\hline Suspeito & 3 & 7,89 \\
\hline Suspeito e Confirmado & 15 & 39,47 \\
\hline Não tive contato & 7 & 18,43 \\
\hline \multicolumn{3}{|l|}{ Treinamento } \\
\hline Sim & 11 & 28,95 \\
\hline Não & 27 & 71,05 \\
\hline Acesso à NT SMSA & 38 & 100,00 \\
\hline \multicolumn{3}{|l|}{ Leitura das NT da SMSA } \\
\hline Sim & 31 & 81,58 \\
\hline Não & 7 & 18,42 \\
\hline \multicolumn{3}{|c|}{ Distanciamento dos familiares } \\
\hline Sim & 33 & 86,84 \\
\hline Não & 5 & 13,16 \\
\hline \multicolumn{3}{|c|}{ Exercício das atividades laborais } \\
\hline Unidade Básica de Saúde & 37 & 97,37 \\
\hline Homeoffice & 1 & 2,63 \\
\hline \multicolumn{3}{|l|}{ Carga horária de trabalho } \\
\hline Manteve & 35 & 92,11 \\
\hline Aumentou & 1 & 2,63 \\
\hline Diminuiu & 2 & 5,26 \\
\hline
\end{tabular}

NT: nota técnica; SMSA: Secretaria Municipal de Saúde; ${ }^{*}(n=30)$

Elaborado pelas autoras.

No exercício das atividades da rotina de trabalho durante a pandemia da SARSCoV-2, $89,47 \%$ relatam acesso a álcool $70 \%$, seguido de máscara cirúrgica $(86,84 \%)$, luvas $(63,16 \%)$ e capotes descartáveis $(63,16 \%)$ - Gráfico 1. 
Gráfico 1 - Acesso aos Equipamentos de Proteção Individual. Belo Horizonte, 2020.

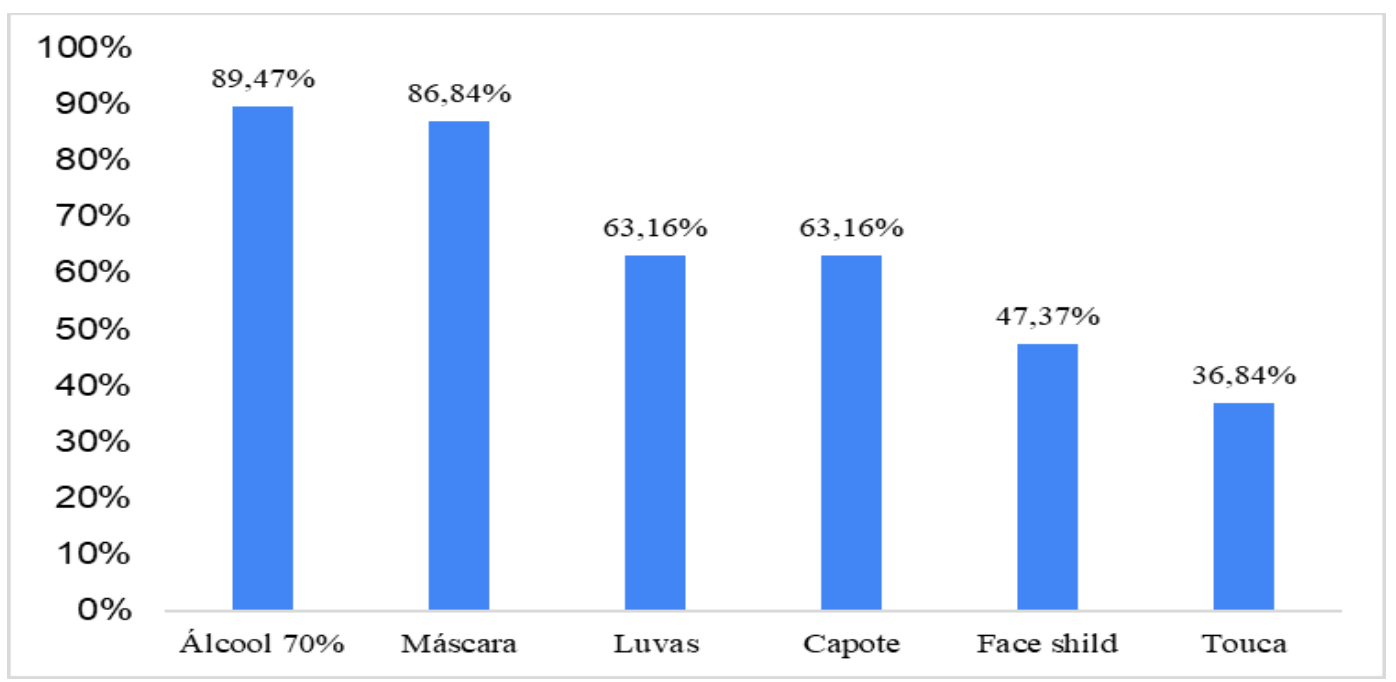

Fonte: Elaborado pelas autoras.

Os atendimentos individuais foram mantidos $(89,47 \%)$, visitas domiciliares (73,68\%), atendimento compartilhado (36,84\%) e reuniões de matriciamento (34,21\%). Já as atividades coletivas, como os grupos operativos, foram totalmente suspensas. Os profissionais realizaram outras atividades como: elaboração de materiais de apoio, protocolos e outras ações de educação permanente.
Para realizar o aconselhamento/orientação aos usuários, durante a pandemia, os profissionais relataram que poderiam ser por: ligação telefônica (84,20\%), seguido de material impresso (57,9\%), uso do aplicativo WhatsApp $(55,30 \%)$, e $31,6 \%$ relataram somente o atendimento presencial como possibilidade Gráfico 2. Porém, 52,63\% relataram que o local de trabalho não possuía tais meios de comunicação.

Gráfico 2 - Meios de comunicação para aconselhamento/orientação. Belo Horizonte, 2020.

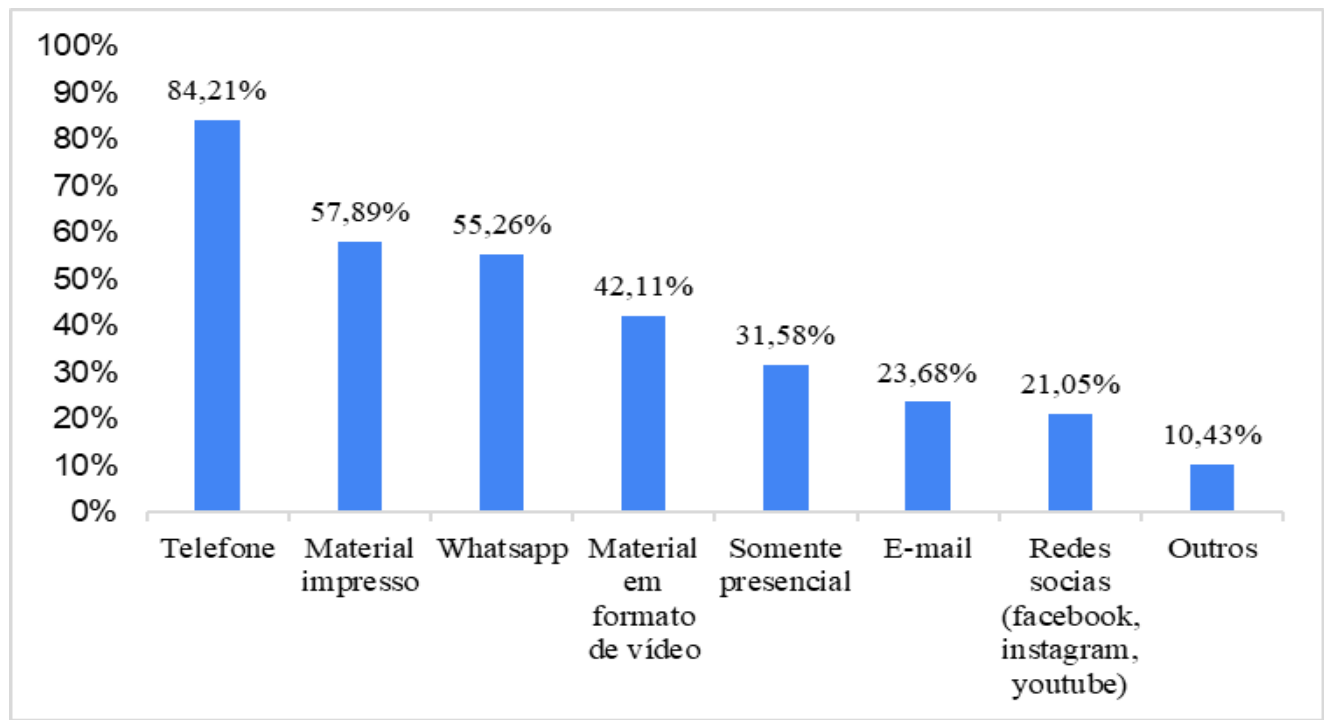

Fonte: Elaborado pelas autoras. 


\section{DISCUSSÃO}

Esta pesquisa revelou que apesar dos profissionais do NASF-AB terem tido contato com casos confirmados e/ou suspeitos da COVID-19, a maioria testou negativo no período estudado. Entretanto, houve relato de não participação em treinamentos para atuar na pandemia e dificuldades para realizar o acompanhamento dos usuários por meios de comunicação não presenciais.

A baixa taxa de contaminação dos profissionais pode ter sido pela conscientização quanto às medidas de biossegurança, como o uso correto dos EPI's, constante higienização das mãos e desinfecção de superfícies; baixo atendimento direto a pessoas infectadas, devido às características intrínsecas das atribuições dos profissionais do NASF-AB. No distrito avaliado há cerca de 57 bairros e até 20 novembro de 2020 foram registrados na região aproximadamente um acumulado de 933 casos confirmados de Síndrome Respiratória Aguda Grave (Boletim Epidemiológico e Assistencial COVID-19 no 155/2020). Além disso, pode ter ocorrido uma baixa testagem para Sars-CoV-2 entre os profissionais, principalmente os assintomáticos.

A observação cotidiana do rápido aumento do número de pessoas infectadas pela doença também pode ter contribuído com uma maior vigilância e autocuidado constante entre estes profissionais. Porém, um estudo realizado no Pará com dados secundários, obtidos diretamente de boletins epidemiológicos disponíveis da página eletrônica da Secretaria Estadual de Saúde do Estado, demonstrou uma alta letalidade entre os profissionais de saúde, o que indica uma maior vulnerabilidade destes (CAMPOS; LEITÃO, 2021).

Os EPI's foram um grande desafio para todos os serviços de saúde e para as diferentes categorias profissionais, tendo em muitos casos impactado na integralidade e longitudinalidade do cuidado (GONÇALVES, 2020; SOARES; PEDUZZI; COSTA, 2020; MUGNATTO, 2020). O medo do contágio levou a um consumo excessivo de EPI na rede assistencial e por parte da população, o que resultou inicialmente em uma situação crítica do sistema para atender a demanda de EPI's (GARCIA et al., 2021; SOARES et al., 2020).

Em virtude disso, em abril foi publicada uma nota técnica municipal com orientações e recomendações sobre o uso racional e seguro de EPI's. Essa nota regulamentou o uso racional explicitando em cada local, como, qual e quando deveriam ser distribuídos e utilizados, garantindo a segurança do profissional e do paciente (GUIMARÃES et al., 2020). Observouse que a maioria dos profissionais teve acesso aos EPI's básicos conforme as recomendações da OMS (OPAS, 2020). Contudo, o uso correto depende da adesão do profissional e treinamentos frequentes são importantes para estimular o uso e reforçar as precauções.

Nessa perspectiva, houve oferta, no município, de capacitação das equipes assistenciais, sobre o funcionamento das UBS's, fluxos de entrada e as atividades eletivas durante a pandemia, além de uma série de capacitações online, incluindo vídeos de treinamento rápido e webconferências (GUIMARÃES et al., 2020). Segundo a OPAS/OMS, é atribuição do gestor de saúde garantir treinamento adequado aos profissionais (OPAS, 2020). Além disso, vários órgãos de ensino a distância disponibilizaram cursos online e gratuitos sobre o manejo da COVID-19, como o Ambiente Virtual de Aprendizagem do Sistema Único de Saúde (AVASUS, 2021), e o Ministério da Saúde (MS) ofertou o Protocolo de Manejo Clínico do Coronavírus (COVID-19) na Atenção Primária à Saúde com o objetivo de qualificar a atuação dos profissionais da APS, garantindo um atendimento resolutivo e a coordenação do cuidado na rede (BRASIL, 2020a).

Contudo, a maioria dos participantes relatou que não participou de treinamentos para atuar na pandemia. Estudo apontou que os profissionais não possuem ou possuem pouco conhecimento sobre o manejo da doença (BARBOSA; SILVA, 2020). Perante essa situação, hipotetiza-se que apesar da disponibilização de capacitações, a participação nos treinamentos pode depender da condução e administração 
dos gerentes das UBS's, além disso, da disponibilidade de equipamentos ou locais para a realização dos treinamentos online ou liberação das horas para realizá-lo no domicílio. No processo de capacitação de equipes, portanto, é importante o alinhamento e troca de informações entre gerentes e profissionais, bem como a sensibilização dos gerentes e a disponibilização de local, equipamentos e horário protegido na agenda dos profissionais.

De fato, os serviços de saúde precisam sistematizar e informatizar o serviço, além de capacitar os profissionais a operá-los, mas também é importante avaliar se os usuários dispõem destes meios para que os atendimentos sejam feitos de maneira a respeitar a integralidade, longitudinalidade e a equidade do cuidado. $O$ uso de tecnologias de informação e comunicação, como WhatsApp e telefone, para a realização de teleconsulta, garante a oferta de ações de forma segura, de modo que não haja descontinuidade e agravamento das condições dos usuários em tratamento (MEDINA, et al., 2020).

Em Florianópolis, observou-se que a pandemia intensificou as mudanças no processo de trabalho com o uso de tecnologias de comunicação (telefone, internet, Whatsapp, vídeo chamada), mas considerando as características da população e do território, sem desassistir aqueles que não utilizam tais tecnologias (SILVEIRA; ZONTA, 2020). Já em Recife, o NASF-AB, manteve sua atuação apoiando as eSF no acolhimento, triagem dos usuários, organização dos fluxos, monitoramentos dos casos sintomáticos. E o vínculo entre as equipes foi considerado como um facilitador na adaptação no processo de trabalho destes profissionais (OLIVEIRA, et al., 2020). Ressalta-se que em Belo Horizonte havia a possibilidade de consulta online para o acompanhamento dos usuários, mas aqueles que não possuíam acesso ou alguma dificuldade na operacionalização dos meios eletrônicos, tinham acesso ao serviço de saúde garantido na UBS de forma segura, minimizando os riscos tanto para o usuário quanto para o profissional.
As atividades de rotina da $A B$ precisam ser preservadas em tempos de pandemia, pois as previsões apontam para um longo curso de convivência com o novo vírus, com alternância de maior e menor isolamento social, o que exige readequação de certos procedimentos $\mathrm{e}$ incorporação de outros para que a $A B$ funcione cumprindo sua missão, incluindo novas formas de cuidado cotidiano à distância, evitando o risco de aprofundamento da exclusão do acesso e das desigualdades sociais (MEDINA, et al., 2020).

Um ponto importante a ser destacado refere-se a doenças infecciosas de alto contágio que necessitam de respostas rápidas do sistema de saúde. No entanto, um sistema historicamente subfinanciado, apresentando congelamento dos gastos públicos por meio da Emenda Constitucional (EC) 95, manifesta dificuldades em enfrentar, com eficiência, o cenário de pandemia descrito. Somado a isto, observa-se alocação de recursos financeiros em maior volume para os planos de saúde, em detrimento ao valor repassado ao SUS (PAIM, et al. 2011; MARTUFI et al., 2020; MATTA et al., 2021).

Considerando ainda aspectos relacionados ao financiamento do SUS, o programa Previne Brasil, instituído em 2019, se apresenta como um novo modelo de financiamento da Atenção Primária à Saúde (APS). Sendo, neste novo modelo, definido pelo gestor local a presença do NASF-AB no município. Isto fragiliza a assistência à saúde de qualidade, que atende as demandas complexas do território, e diminui a capacidade resolutiva da APS, pois interrompe a operacionalização do processo de trabalho compartilhado e interdisciplinar do NASF-AB, contrária à assistência médico-centrada (BRASIL, 2020b; MASSUDA, 2020; REIS; MENESES, 2020).

Considerando o contexto atual, é necessário refletir sobre uma política de acesso, para que os serviços de saúde tenham os meios necessários para a continuidade dos atendimentos aos usuários, telefones, computadores, câmera web, microfones, de forma efetiva para garantir a integridade, 
segurança e sigilo dos dados e informações nas consultas online. Ademais, em eventos como a pandemia, principalmente para os grupos de risco poderiam ser criadas planilhas/programas com alimentação diária de parâmetros importantes a serem observados promovendo a continuidade da atenção em saúde e do cuidado e oportunizando ação quando necessário.

Assim, observou-se que algumas mudanças são necessárias para aprimorar o desempenho da equipe, como o aperfeiçoamento da capacitação dos profissionais, maior valorização do profissional, assim como o investimento na melhoria da infraestrutura de trabalho. Sabemos que uma equipe multiprofissional é uma potência para os serviços de saúde e o NASF-AB pode contribuir com as atividades das eSF através de notificação/monitoramento de casos de COVID-19, apoio/suporte através da avaliação dos pacientes crônicos, campanhas de vacinação e saúde do trabalhador (acolhendo, incentivando o autocuidado e escuta dos profissionais das UBS's).

É importante destacar que o processo de trabalho dos profissionais é dinâmico, com alterações constantes, variando conforme os indicadores da COVID-19. Existem limitações quanto à metodologia (websurveys) e períodos estudados, visto a rápida dinâmica da pandemia, o que contribui as limitações específicas. Uma delas é a cobertura e/ou acesso de internet pelos profissionais. A falta de representatividade é a principal limitação de nosso estudo, portanto, não é possível generalizar os resultados, mas os achados são importantes visto que a pandemia da COVID-19 afetou todos os serviços de saúde. Além disso, todos os dados coletados são autorrelatados e isso pode torná-los de baixa confiabilidade, especialmente quando se trata de relatar processo de trabalho para os quais pode haver subnotificação ou superestimação, mesmo garantido o anonimato e a confidencialidade. Outra limitação é a possibilidade de viés de auto-seleção, pois profissionais de saúde sem confirmação de infecção podem ter maior probabilidade de participar do estudo, visto o estigma de ser saudável.
Por fim, este estudo permitiu discorrer sobre as principais facilidades e dificuldades presentes no trabalho do NASF-AB em uma regional do município, durante a pandemia pelo Sars-CoV2. É imperativo destacar que com todas as dificuldades enfrentadas, o NASF-AB possui profissionais que podem exercer suas atividades e serviços no enfrentamento em situações semelhantes à COVID-19, como na notificação, vigilância dos casos, orientação, continuidade do cuidado e educação em saúde.

\section{CONSIDERAÇÕES FINAIS}

De forma geral, os achados deste estudo identificaram fragilidades em relação à falta de equipamentos tecnológicos e de informática anteriores à pandemia da COVID-19 e a baixa participação de capacitações para execução do trabalho do NASF-AB durante a pandemia. É necessário reconhecer as adversidades do processo de trabalho e garantir investimentos necessários para que as equipes executem suas atividades de forma efetiva em diversas situações.

Diante dos desafios nas atividades das equipes, onde a situação exigiu superação das limitações e mudanças de comportamentos e hábitos, tanto dos profissionais quanto dos usuários, e paralelamente a promoção de um atendimento qualificado, colaborativo e interdisciplinar, o NASF-AB tem um papel muito importante nos territórios. 


\section{REFERÊNCIAS}

AVASUS. Ambiente Virtual de Aprendizagem do Sistema Único de Saúde. Disponível em: https://avasus.ufrn.br/. Acesso em 16 de dezembro de 2021.

BRASIL. Ministério da Saúde. Secretaria de Atenção à Saúde. Portaria n 154, de 24 de janeiro de 2008. Cria os Núcleos de Apoio à Saúde da Família - NASF. Diário Oficial da União, Brasília, DF, 2008. Disponível em: http://bvsms.saude.gov.br/bvs/saudelegis/gm/2008/prt0154_24_01_2008.html>. Acesso em 01 de maio 2020.

BRASIL. Ministério da Saúde. Secretaria de Atenção à Saúde. Departamento de Atenção Básica. Núcleo de Apoio de Saúde da Família: Ferramenta para a gestão e para o trabalho cotidiano. Caderno 39, Brasília, DF, 2014.2 Disponível em: http://bvsms.saude.gov.br/bvs/publicacoes/caderno_atencao_basica_diretrizes_nasf.pdf. Acesso em 1 de março de 2020.

BRASIL. Organização Pan-Americana de Saúde. Uso Racional de Equipamentos de proteção individual para a doença causada pelo coronavírus 2019 (COVID-19). Disponível em: https://iris.paho.org/bitstream/handle/10665.2/51954/OPASBRACOVID1920013_por.pdf?sequence=1\& isAllowed=y . Acesso em 11 de janeiro de 2021.

BRASIL. Ministério da Saúde. Painel Coronavírus. Brasília, DF, 2021. Disponível em: https://covid.saude.gov.br/. Acesso em 15 de julho de 2021.

BRASIL. Ministério da Saúde. Secretaria de Atenção Primária à Saúde. Protocolo de Manejo Clínico do Coronavírus (COVID-19) na Atenção Primária à Saúde - Versão 9. Brasília, DF, 2020a. Disponível em: https://docs.bvsalud.org/biblioref/2020/05/1095920/20200504-protocolomanejo-ver09.pdf. Acesso em 16 de dezembro de 2021.

BRASIL. Ministério da Saúde. Secretaria de Atenção Primária à Saúde Departamento de Saúde da Família. NOTA TÉCNICA № 3/2020-DESF/SAPS/MS. Brasília, DF, 2020b. Disponível em: https://www.conasems.org.br/wp-content/uploads/2020/01/NT-NASF-AB-e-Previne-Brasil-1.pdf. Acesso em 16 de dezembro de 2021.

BARBOSA, S. DE P.; SILVA, A. V. F. G. A Prática da Atenção Primária à Saúde no Combate da Covid-19. APS em Revista, v.2, n.1, p.17-19, 2020. Disponível em: https://apsemrevista.org/aps/article/view/62/43. Acesso em 25 de maio de 2021.

CAMPOS, A. C. V.; LEITÃO, L. P. C. Letalidade da COVID-19 entre profissionais de saúde no Pará. Journal Health NPEPS, v.6, n.1, p.22-34, 2021.

FERNANDEZ, M. V. et al. Reorganizar para avançar: a experiência da Atenção Primária à Saúde de Nova Lima/MG no enfrentamento da pandemia da COVID-19. APS em Revista, v. 2, n. 2, p.114-121, 2020.

FILHO, J. M. J. et al. A Saúde do Trabalhador e o Enfrentamento da COVID-19. Revista Brasileira de Saúde Ocupacional, v.45, n.14, $2020 . \quad$ Disponível em: https://www.scielo.br/j/rbso/a/Km3dDZSWmGgpgYbjgc57RCn/?lang=pt\&format=pdf. Acesso em 25 de maio de 2021.

GARCIA, G. P. A. et al. Utilização de equipamentos de proteção individual para atendimento de pacientes com covid-19: revisão de escopo. Revista Gaúcha de Enfermagem, V.42, 2021.

GONÇALVES, N. C. P. Trabalho em saúde na Atenção Primária no contexto de pandemia: novos instrumentos de trabalho podem possibilitar rupturas e transformações? Journal of Management Primary Health Care, v.12, $2020 . \quad$ Disponível em: https://www.jmphc.com.br/jmphc/article/view/1028/950. Acesso em 16 de dezembro de 2021.

GUIMARÃES, F. G. et al. A Organização da Atenção Primária à Saúde de Belo Horizonte no Enfrentamento da Pandemia COVID-19: Relato de Experiência. APS em Revista, v. 2, n. 2, p. 74-82, 2020. Disponível em: https://apsemrevista.org/aps/article/view/128/55. Acesso em 11 de maio de 2021. 
MACIEL, M. DOS. S. et al. Ações de Saúde Desenvolvidas pelo Núcleo de Apoio à Saúde da Família NASF. Revista Saúde Santa Maria, v. 41, n.1, p.117-122, 2015. Disponível em: https://periodicos.ufsm.br/revistasaude/article/view/13283/pdf. Acesso em 7 de junho de 2021.

MARTUFI, V. et al. Desafios da APS no SUS no enfrentamento da Covid-19. Apresentação do Seminário Virtual Rede APS Abrasco, 2 abr. 2020. Salvador, Belo Horizonte, Rio de Janeiro. Disponível em: https://redeaps.org.br/wp-content/uploads/2020/04/Relatorio-Rede-APS-_Seminário-APS-no-SUS-eCovid-16-Abril-2020-final.pdf. Acesso em 16 de dezembro de 2021.

MASSUDA, A. Mudanças no financiamento da Atenção Primária à Saúde no Sistema de Saúde Brasileiro: avanço ou retrocesso? Ciência \& Saúde Coletiva, v. 25, n. 4, 2020.

MATTA, G. C. et al. Os impactos sociais da Covid-19 no Brasil: populações vulnerabilizadas e respostas à pandemia [online]. Rio de Janeiro: Observatório Covid 19; Editora FIOCRUZ, P. 221, 2021. Informação para ação na Covid-19 series. ISBN: 978-65-5708-032-0. Acesso em 16 de dezembro de 2021.

MEDINA, M. G. et al. Atenção Primária à Saúde em tempos de COVID-19: O que Fazer? Caderno de Saúde Pública, $\quad$ v. 36, n.8, 2020. Disponível em: https: http://cadernos.ensp.fiocruz.br/static/arquivo/1678-4464-csp-36-08-e00149720.pdf. Acesso em 7 de junho de 2021.

MUGNATTO, S. Agentes comunitários de saúde reclamam da falta de equipamentos de proteção. Agência Câmara de Notícias. 2 jun. 2020. Disponível em: https://www.camara.leg.br/noticias/666263agentes-comunitarios-de-saude-reclamam-da-falta-de-equipamentos-de-protecao/. Acesso em 16 de dezembro de 2021.

OLIVEIRA, M. A. DE B. et al. A Prática do Núcleo de Apoio à Saúde da Família do Recife no Enfrentamento à Pandemia COVID-19. APS em Revista, v. 2, n. 2, p. 142-150, 2020. Disponível em: https://apsemrevista.org/aps/article/view/96/64. Acesso em 16 de junho de 2021.

ORGANIZAÇÃO PAN-AMERICANA DA SAÚDE - OPAS. Uso racional de equipamentos de proteção individual para a doença causada pelo coronavírus 2019 (COVID-19). Orientação provisória, 27 de fevereiro de 2020. Disponível em: https://iris.paho.org/handle/10665.2/51954. Acesso em 16 de dezembro de 2021.

PAIM, J. et al. O sistema de saúde brasileiro: história, avanços e desafios. Saúde no Brasil 1. The Lancet, 2011. Disponível em: https://actbr.org.br/uploads/arquivo/925_brazil1.pdf. Acesso em 16 de dezembro de 2021.

PREFEITURA MUNICIPAL DE BELO HORIZONTE. Boletim Epidemiológico e Assistencial COVID-19 no 155/2020. Disponível em: https://prefeitura.pbh.gov.br/saude/coronavirus. Acesso em 16 de dezembro de 2021.

RAFAEL, R. M. R. et al. Epidemiologia, Políticas Públicas e Pandemia de Covid-19 : o que esperar no Brasil ? Revista Enfermagem UERJ, v. 28, p. 2-7, 2020. Disponível em: https://www.epublicacoes.uerj.br/index.php/enfermagemuerj/article/view/49570/33134. Acesso em 16 de junho de 2021.

REIS, S.; MENESES, S. Novo Financiamento da Atenção Básica: possíveis impactos sobre o NASF-AB. Centro Brasileiro de Estudos em Saúde (Cebes), 12 fev. 2020. Disponível em: https://cebes.org.br/novofinanciamento-da-atencao-basica-impactos-sobre-o-nasf-ab/21242/. Acesso em 16 de dezembro de 2021.

RIBEIRO, M. A. et al. (RE)Organização da Atenção Primária à Saúde para o enfrentamento da COVID-19: Experiência de Sobral-CE. APS em Revista, v. 2, n. 2, p. 177-188, 2020.

SILVEIRA, J. P. M.; ZONTA, R. Experiência de Organização da APS para o Enfrentamento da COVID-19 em Florianópolis. APS em Revista, v. 2, n. 2, p. 91-96, 2020. Disponível em: https://apsemrevista.org/aps/article/view/122/57. Acesso em 21 de junho de 2021. 
SOARES, C.B.; PEDUZZI, M.; COSTA, M. V. Nursing workers: Covid-19 pandemic and social inequalities. Revista da Escola de Enfermagem da USP, v. 54, 2020. Disponível em: https://www.scielo.br/j/reeusp/a/TkQMY6gqJnXwpRzkGQN8V6P/?lang=en. Acesso em 16 de dezembro de 2021.

SOARES, S. S. S. et al. Covid-19 e uso racional de EPI. Revista Enfermagem UERJ, v. 28, 2020.

WERNECK, G. L.; CARVALHO, M. S. A Pandemia de COVID-19 no Brasil: Crônica de uma Crise Sanitária Anunciada. Caderno de Saúde Pública, v.36, n.5, 2020. Disponível em: https://www.scielosp.org/pdf/csp/2020.v36n5/e00068820/pt. Acesso em 16 de junho de 2021.

WORLD HEALTH ORGANIZATION - WHO. Director-General's opening remarks at the media briefing on COVID-19 - 11 March 2020. Disponível em: https://www.who.int/director-general/speeches/detail/whodirector-general-s-opening-remarks-at-the-media-briefing-on-covid-19---11-march-2020. Acesso em 16 de dezembro de 2021. 